\title{
The danger of mercury vapours from aspirators
}

\author{
Mercury vapour release from a dental aspirator by C. A. Stonehouse and A. P. Newman Br Dent J 2001; 190:558-560
}

\begin{abstract}
Objective
To investigate the release of mercury vapour from a dental aspirator which vented its waste air through its base directly into the surgery environment.

Methodology

Mercury vapour in air concentrations were measured at the breathing zone of the dentist during continuous operation of the aspirator. Further series of mercury vapour measurements taken at the aspirator exhaust vent were carried out to determine the sources of mercury vapour from this particular device.
\end{abstract}

\section{Results \\ At the dentist's breathing zone, mercury vapour concentrations of ten times the current occupational exposure limit of $25 \mu \mathrm{g} / \mathrm{m}^{3}$ were recorded after 20 minutes of continuous aspirator operation. A build up of amalgam contamination within the internal corrugated tubing of the aspirator was found to be the main source of mercury vapour emissions followed by particulate amalgam trapped within the vacuum motor. As the vacuum motor heated up with run time, mercury vapour emissions increased. It was found that the bacterial air exhaust filter (designed to clean the contaminated waste air entering the surgery) offered no protection to mercury vapour. In this case the filter trapped particulate amalgam which contributed to further}

mercury vapour contamination as high volume air was vented through it.

\section{Conclusion \\ It is not known how many dental aspirators are in use that vent their waste air directly into the surgery or if this aspirator is representative of others in existence. The safety of dental aspirating systems with regard to mercury vapour exposure requires further investigation.}

\section{Comment}

The risk of mercury exposure from for dentists than patients because of their continuous exposure over their working day. The main risks of mercury exposure from amalgam usage occur from mercury spillage, amalgam particle contamination of the surgery, exposure during amalgam placement, removal or cutting, release from mechanical triturators and release from dental aspirators. The risks from a number of these sources are reduced by the use of encapsulated amalgam. However, this still leaves the dental aspirator as a major risk of contamination. This is because it can become progressively contaminated with amalgam during use and because it heats up during running since this is known to markedly increase mercury release. A number of studies of dentists and surgeries in the past have shown that many surgeries become contaminated and this can result in mercury absorption by dentists and their staff. This can be shown by mercury measurements of blood, urine and hair. Most of these risks can be markedly reduced by good mercury hygiene practice as set out in the BDA guidelines. Furthermore serial studies in the USA at annual ADA meetings have shown that the urine mercury levels in dentists reduced from an average of $14.2 \mu \mathrm{g} / \mathrm{L}$ in 1975 to $6.7 \mu \mathrm{g} / \mathrm{L}$ in 1985 to $4.94 \mu \mathrm{g} / \mathrm{L}$ in 1995 . These levels should be seen in the context that reduced neurobehavioural performance can occur in some individuals with urine mercury levels above $20 \mu \mathrm{g} / \mathrm{L}$ and also that on the basis of industrial studies that levels in the urine below $15 \mu \mathrm{g} / \mathrm{L}$ are considered safe.

This study highlights a number of important issues with regard to aspirators. The first is the danger of buying second hand equipment which may already be badly contaminated. The second is the issue of waste air exhaust. This model, like most dental aspirators, vented into the surgery air resulting in major air contamination. The latest version of this aspirator and other similar equipment have the option to vent into the outside air and this is obviously preferable. Thirdly it highlights the need to regularly clean the internal and external pipe work and change the air filters of such equipment. It is also important to protect the airways of individuals performing this task by wearing a mercury vapour absorbent mask and by waiting until the equipment has fully cooled before under- taking this work. Furthermore, such work should not be carried out by pregnant females as mercury vapour can cross the placenta. Finally, all waste amalgam should be regularly removed from the collection canister and appropriately stored. Good care should be taken to avoid contamination of the surgery waste pipes with waste amalgam since this will contaminate sewage and hence the environment. Amalgam contamination of the waste water is best avoided by careful hygiene and the fitting of an amalgam separator. These devices have been shown to greatly reduce mercury emissions by this route.

If the decision is made to vent the aspirator air exhaust to the outside it should also be noted that this can contaminate the environment but to a much lesser degree since there is a massive reduction in its concentration by the large volume of outside air. It should also be noted that this study highlights the need for good surgery ventilation to prevent any build up of mercury vapour.

\section{Barry Eley}

Professor, Department of Periodontology,

GKT Dental Institute 